\title{
ON THE HETEROTIC/F-THEORY DUALITY IN EIGHT DIMENSIONS
}

\author{
W. Lerche \\ CERN, Geneva, Switzerland
}

\begin{abstract}
We review quantitative tests on the duality between the heterotic string on $T^{2}$ and $F$-theory on $K 3$. On the heterotic side, certain threshold corrections to the effective action can be exactly computed at one-loop order, and the issue is to reproduce these from geometric quantities pertaining to the $K 3$ surface. In doing so we learn about certain nonperturbative interactions of 7-branes.
\end{abstract}

\section{INTRODUCTION}

One of the most basic dualities in string theory is the one between the heterotic string, compactified on the two-torus $T^{2}$, and $F$-theory on $K 3$ [1]; indeed other dualities can be derived from this duality in $d=8$. In fact, the higher the uncompactified space-time dimension is, the simpler the structure of non-perturbative effects becomes, and related to that, the less complicated the structure of the moduli space is. As we will see, in $d=8$ the structure is simple enough for exactly computing certain non-perturbative quantities, but still complicated enough to obtain functionally non-trivial results.

The point is that certain pieces of the effective action can be computed exactly at one-loop order in the heterotic formulation. This is the reason why this model provides an ideal framework for studying non-trivial brane interactions; in the past, it has been very successfully applied to study brane interactions in type I and matrix strings (see eg., [2, 3, 4]). Our aim, on the other hand, is to show how these coupling functions can be reproduced in $F$-theory, ie., from $K 3$ geometry. This has also a direct interpretation in terms of certain Type IIB $D$-brane interactions, which gives another motivation for studying this subject.

Usually, when studying interactions between $D$-branes, one considers idealized situations where one focuses one a single pair of (possibly stacks 
of) branes. In addition, these branes are usually mutually "local", i.e., they can be simultaneously described at weak coupling, and so treated with methods of conformal field theory.

However, for making quantitative tests of string dualities involving highly non-trivial functions, the full global structure of $D$-brane interactions becomes important as well - that is, the influence of all the other branes that are usually considered as far away. Some of the branes are necessarily non-local with respect to each other, which precludes any conformal field theoretic treatment of the full theory.

Therefore, in order to exactly determine the functional dependence of the relevant interactions, new non-perturbative methods are called for. Guided by the exact results that can be obtained in the heterotic formulation, we will show how these interactions can be described in geometrical terms. This approach has been presented in refs. [5, 6, 7], and this is what we will -partially- review from section 3.4 onwards.

However, before we will come to that, we will first review some simple facts about the structure of the coupling functions under consideration. Subsequently we will present a brief introduction to the relevant aspects of $F$-theory.

\section{BPS SATURATED, EXACT HETEROTIC AMPLITUDES AT ONE-LOOP ORDER}

One certainly cannot expect to compute any given piece of the effective lagrangian exactly. It is in general just very special couplings, namely typically those which are anomaly-related and to which only BPS-states contribute, that are amenable to an exact treatment. In the present situation with 16 supercharges in eight dimensions, the canonical BPS-saturated amplitudes [8, 9] involve four external gauge bosons (and/or gravitons that we will not consider here).

Supersymmetry relates parity even $\left(i \xi F^{n}\right)$ and parity odd $\left(\frac{\theta}{2 \pi} F \wedge\right.$ $F \wedge . . F)$ sectors, and one can conveniently combine the theta-angle and the coupling constant $\xi$ into one complex coupling, $\Delta_{\text {eff }}$. In particular, when compactifying the heterotic string on $T^{2}$, the effective threshold couplings $\Delta_{\text {eff }}(T, U) \equiv i \xi(T, U)+\frac{1}{2 \pi} \theta(T, U)$ become highly non-trivial functions depending on the usual torus Kähler and complex structure moduli, $T$ and $U$.

As mentioned before, in the heterotic string picture these couplings are exact at one-loop order; this is simply because there are no instantons that could possibly contribute (apart from the world-sheet instantons whose effects are captured in the one-loop computation). The couplings are in fact directly related [8] to the heterotic elliptic 
genus [10], which is given by the Ramond partition function in the presence of a non-vanishing background gauge field strength, roughly: $\widehat{\mathcal{A}}(F, q) \sim \operatorname{Tr}_{R}(-1)^{J_{0}} q^{L_{0}} e^{F}$. More precisely, the couplings are typically given by modular integrals of the form:

$$
\left.\operatorname{Re}\left[\Delta_{\mathrm{eff}}(T, U)\right] F \wedge \ldots F \sim \int \frac{d^{2} \tau}{\tau_{2}} Z_{(2,2)}(T, U, q) \widehat{\mathcal{A}}(F, q)\right|_{8-\text { form }}
$$

where $Z_{(2,2)}$ is the partition function of the two-torus $T^{2}$ :

$$
Z_{2,2}(T, U, q)=\sum_{p_{L}, p_{R}} q^{\frac{1}{2}\left|p_{L}\right|^{2}} \bar{q}^{\frac{1}{2}\left|p_{R}\right|^{2}}
$$

with $p_{L}=\frac{1}{\sqrt{2 T_{2} U_{2}}}\left(m_{1}+m_{2} \bar{U}+n_{1} \bar{T}+n_{2} \bar{T} \bar{U}\right), p_{R}=\frac{1}{\sqrt{2 T_{2} U_{2}}}\left(m_{1}+m_{2} \bar{U}+\right.$ $n_{1} T+n_{2} T \bar{U}$ ) (we switched off the Wilson lines here). The evaluation of modular integrals of type (1.1) is quite an art that has been discussed at length in $[11,3,5,6]$, but won't be touched upon here. Essentially, each of such integrals results in a certain holomorphically factorized, Borcherds product type of automorphic function:

$$
\begin{aligned}
\Delta_{\text {eff }}(T, U) & =\ln [\Psi], \quad \text { where } \\
\Psi & =\left(q_{T}\right)^{a}\left(q_{U}\right)^{b} \prod_{(k, l)>0}\left(1-q_{T}^{k} q_{U}\right)^{c(k l)},
\end{aligned}
$$

for some $a, b$. Here, $q_{T}=e^{2 \pi i T}, q_{U}=e^{2 \pi i U}$, the product runs over $k>0, l \in \mathbb{Z} \wedge k=0, l>0$ in the chamber $T_{2} \equiv \operatorname{Im} T>U_{2} \equiv \operatorname{Im} U$, and $c(n)$ are the expansion coefficients of a certain nearly holomorphic and (quasi-)modular form, $\mathcal{C}(q) \equiv \sum c(n) q^{n}$. The precise form of this "counting function" $\mathcal{C}(q)$ depends on the specific gauge couplings that are considered.

Specifically, if we switch off all the Wilson lines so that we have $E_{8} \times E_{8}^{\prime}$ non-abelian gauge symmetry, we have $[3,6]$ :

$$
\begin{array}{r}
\Delta_{E_{8} E_{8}^{\prime}}(T, U)=-48 \ln [\Psi], \text { with } a=-2, b=0 \text { and counting function } \\
\qquad \begin{aligned}
\mathcal{C}(q) & =\frac{1}{12} \frac{1}{\eta^{24}}\left[E_{2} E_{4}-E_{6}\right]^{2}(q) \\
\Delta_{E_{8} E_{8}}(T, U)=-24 \ln [\Psi], \text { with } a & =8, b=12 \text { and counting function } \\
\mathcal{C}(q) & =\frac{1}{12} \frac{E_{4}}{\eta^{24}}\left[E_{2}^{2} E_{4}-2 E_{2} E_{6}+E_{4}^{2}\right](q),
\end{aligned}
\end{array}
$$

where $E_{n}(q)$ are the usual Eisenstein series of the corresponding modular weight. Moreover, for those couplings for which the field strengths $F=\left\{F_{T}, F_{U}\right\}$ are superpartners of the torus moduli, an extra structure 
$[5,7]$ emerges: namely these couplings satisfy non-trivial integrability conditions and so can be obtained as fourth derivatives of the following holomorphic prepotential:

$$
\mathcal{G}(T, U) \sim \sum_{(k, l)>0} c(k l) \mathcal{L} i_{5}\left(q_{T}^{k} q_{U}^{l}\right),
$$

with counting function given by $\mathcal{C}(q)=\frac{E_{4}^{2}}{\eta^{24}}(q)$. Above, the polylogarithm is defined by $\mathcal{L} i_{a}(z)=\sum_{p>0} \frac{z^{p}}{p^{a}}(a \geq 1)$.

An physically interesting feature of these couplings in the $T, U$ subsector is that they have logarithmic singularities, consider for example:

$$
\begin{aligned}
\Delta_{\mathrm{eff}}^{(T T U U)}(T, U) & \equiv\left(\partial_{T}\right)^{2}\left(\partial_{U}\right)^{2} \mathcal{G}(T, U) \\
& =\frac{1}{2 \pi i} \ln [J(T)-J(U)]+\frac{1}{2 \pi i} \ln \left[\Psi_{0}(T, U)\right],
\end{aligned}
$$

where $\Psi_{0}(T, U)$ is some cusp form that stays finite over the whole of the moduli space. Similar to the analogous situation in four dimensions $[12,13]$, the modular invariant $J$-functions encode the gauge symmetry enhancements pertaining to the compactification torus $T^{2}: S U(2)$ for $T=U, S U(2) \times S U(2)$ at $T=U=i$ and $S U(3)$ at $T=U=\rho \equiv$ $e^{2 \pi i / 3}$, and in particular reflect the charge multiplicities of the states becoming light near the singularities. Specifically, near the $S U(2)$ locus the coupling behaves like:

$$
\Delta_{\mathrm{eff}}^{(T T U U)}(T, U) \sim \ln \left[\sqrt{\alpha^{\prime}} a\right], \quad a \equiv \frac{1}{\sqrt{\alpha^{\prime}}}(T-U),
$$

and similar for the other gauge groups. This is the expected behavior of the one-loop field theory effective action, with cutoff scale given by $\alpha^{\prime}$.

The issue is to reproduce the threshold coupling functions $\Delta_{\text {eff }}(T, U)$ in the dual $F$-theory compactification on $K 3$ (we will actually consider one-dimensional slices of the moduli space with constant $U$ ). For this, we will first briefly review some of the relevant basic features on $F$-theory that we will need.

\section{REVIEW OF F-THEORY \\ 3.1 ELLIPTIC FIBRATIONS}

$F$-theory compactifications [1] are by definition compactifications of the type IIB string with non-zero, and in general non-constant string

\footnotetext{
${ }^{1}$ The very existence of a holomorphic prepotential hints at the existence of a yet unknown superspace formulation of the theory, in which the prepotential would figure as the effective lagrangian.
} 
coupling - they are thus intrinsically non-perturbative. F-theory may also seen as a construction to geometrize (and thereby making manifest) certain features pertaining to the $S$-duality of the type IIB string.

To explain this in somewhat more detail, let us first recapitulate the most important massless bosonic fields of the type IIB string. From the NS-NS sector, we have the graviton $g_{\mu \nu}$, the antisymmetric 2-form field $B$ as well as the dilaton $\phi$; the latter, when exponentiated, serves as the coupling constant of the theory. Moreover, from the $\mathrm{R}-\mathrm{R}$ sector we have the $p$-form tensor fields $C^{(p)}$ with $p=0,2,4$. It is also convenient to include the magnetic duals of these fields, $B^{(6)}, C^{(6)}$ and $C^{(8)}\left(C^{(4)}\right.$ has self-dual field strength). It is useful to combine the dilaton with the axion into one complex field:

$$
\tau_{I I B} \equiv C^{(0)}+i e^{-\phi} .
$$

The $S$-duality then acts via projective $S L(2, \mathbb{Z})$ transformations in the canonical manner: $\tau_{I I B} \rightarrow \frac{a \tau_{I I B}+b}{c \tau_{I I B}+d}$ with $a, b, c, d \in \mathbb{Z}$ and $a d-b c=$ 1. Furthermore, it acts via simple matrix multiplication on the other fields if these are grouped into doublets $\left(\begin{array}{l}B^{(2)} \\ C^{(2)}\end{array}\right),\left(\begin{array}{l}B^{(6)} \\ C^{(4)}\end{array}\right)$ (while $C^{(4)}$ stays invariant).

The simplest $F$-theory compactifications are the highest dimensional ones, and simplest of all is the compactification of the type IIB string on the 2-sphere, $\mathbb{P}^{1}$. $c_{1}\left(\mathbb{P}^{1}\right)=-2$, this by itself cannot be a good, supersymmetry preserving background. The remedy is to add extra 7-branes to the theory, which sit at arbitrary points $z_{i}$ on the $\mathbb{P}^{1}$, and otherwise fill the $7+1$ noncompact space-time dimensions. If this is done in the right way, $c_{1}\left(\mathbb{P}^{1}\right)$ is cancelled, thereby providing a consistent background.

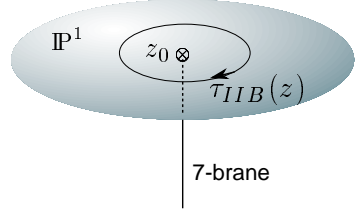

Figure 1.1 Encircling the location of a 7-brane in the z-plane leads to a jump of the perceived type IIB string coupling, $\tau_{I I B} \rightarrow \tau_{I I B}+1$.

To explain how this works, consider first a single $D 7$-brane located at an arbitrary given point $z_{0}$ on the $\mathbb{P}^{1}$. A $D 7$-brane carries by definition

${ }^{2}$ Six and in particular for dimensional compactifications are much more complicated than the eight dimensional one discussed here, and any discussion of them would be beyond the scope of this lecture. 
one unit of D7-brane charge, since it is a unit source of $C^{(8)}$. This means that is it magnetically charged with respect to the dual field $C^{(0)}$, which enters in the complexified type IIB coupling in (1.6). As a consequence, encircling the plane location $z_{0}$ will induce a non-trivial monodromy, that is, a jump on the coupling - see Fig. 1.1. But this then implies that in the neighborhood of the $D 7$-brane, we must have a non-constant string coupling of the form: $\tau_{I I B}(z)=\frac{1}{2 \pi i} \ln \left[z-z_{0}\right]$; we thus indeed have a truly non-perturbative situation.

In view of the $S L(2, \mathbb{Z})$ action on the string coupling (1.6), it is natural to interpret it as a modular parameter of a two-torus, $T^{2}$, and this is what then gives a geometrical meaning to the $S$-duality group [1]. Since, as we have seen, this modular parameter $\tau_{I I B}=\tau_{I I B}(z)$ is not constant over the $\mathbb{P}^{1}$ compactification manifold, the shape of the $T^{2}$ will accordingly vary along $\mathbb{P}^{1}$. The relevant geometrical object will therefore not be the direct product manifold $T^{2} \times \mathbb{P}^{1}$, but rather a fibration of $T^{2}$ over $\mathbb{P}^{1}$ see Fig. 1.2.

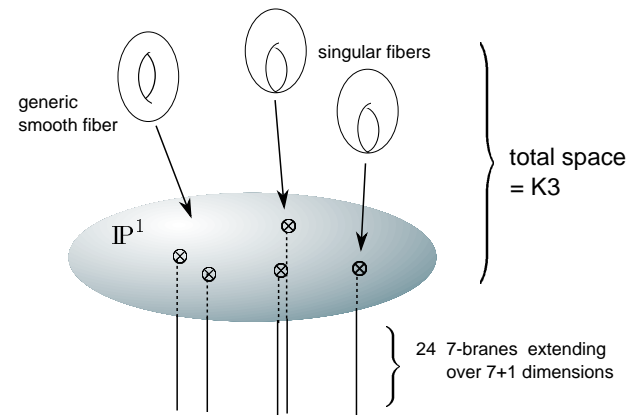

Figure 1.2 Fibration of an elliptic curve over $\mathbb{P}^{1}$, which in total makes a $K 3$ surface. At 24 points the fibers and therefore the string coupling become singular, and this is where the 7-branes are located.

The logarithmic behavior of $\tau_{I I B}(z)$ in the vicinity of a 7-brane means that the $T^{2}$ fiber is singular at the brane location. It is known from mathematics that each of such singular fibers contributes $1 / 12$ to the first Chern class. Therefore we need to put 24 of them in order to have a consistent type IIB background with $c_{1}=0$. The mathematical data: " $T^{2}$ fibered over $\mathbb{P}^{1}$ with 24 singular fibers" is now exactly what characterizes the $K 3$ surface; 3 indeed it is the only complex two-dimensional manifold with vanishing first Chern class (apart from $T^{4}$ ).

${ }^{3}$ More precisely, an "elliptically fibered" $K 3$; we assume here singularities of the simplest canonical type. See ref. [14] for a physicist's review of the $K 3$ manifold. 
The $K 3$ manifold that arises in this context is so far just a formal construct, introduced to encode of the behavior of the string coupling in the presence of 7-branes in an elegant and useful way. One may speculate about a possible more concrete physical significance, such as a compactification manifold of a yet unknown 12 dimensional " $F$-theory" [1]. The existence of such a theory is still unclear, but not really important for our purposes; all we need the $K 3$ for is to use its intriguing geometric properties for computing physical quantities (the quartic gauge threshold couplings, ultimately).

In order to do explicit computations, we first of all need a concrete representation of the $K 3$ surface. Since the families of $K 3$ 's in question are elliptically fibered, the natural starting point is the two-torus $T^{2}$. It can be represented in the well-known "Weierstraß" form:

$$
W_{T^{2}}=y^{2}+x^{3}+x f+g=0,
$$

which in turn is invariantly characterized by the $J$-function:

$$
J=\frac{4(24 f)^{3}}{4 f^{3}+27 g^{2}} .
$$

An elliptically fibered $K 3$ surface can be made out of (1.7) by letting $f \rightarrow f_{8}(z)$ and $g \rightarrow g_{12}(z)$ become polynomials in the $\mathbb{P}^{1}$ coordinate $z$, of the indicated orders. The locations $z_{i}$ of the 7-branes, which correspond to the locations of the singular fibers where $J\left(\tau_{I I B}\left(z_{i}\right)\right) \rightarrow \infty$, are then precisely where the discriminant

$$
\begin{aligned}
\Delta(z) & \equiv 4 f_{8}{ }^{3}(z)+27 g_{12}{ }^{2}(z) \\
& =: \prod_{i=1}^{24}\left(z-z_{i}\right)
\end{aligned}
$$

vanishes.

\subsection{MONODROMIES AND LOCALITY}

Note that the polynomials $f_{8}(z)$ and $g_{12}(z)$ in (1.7) together have exactly 18 independent free parameters. It is indeed a mathematical fact that the moduli space of elliptic $K 3$ manifolds is 18 complex-dimensional and furthermore locally given by

$$
M=\frac{S O(18,2)}{S O(18) \times S O(2)} .
$$

This just happens to be the same as the moduli space of the heterotic string on $T^{2}$ (which includes the torus moduli $T, U$ besides the 16 Wilson lines) ! This coincidence of moduli spaces was one of the primary 
motivations [1] for postulating the duality between $F$-theory on $K 3$ (or type IIB on $\mathbb{P}^{1}$ with 247 -branes) and the heterotic string on $T^{2}$.

However, (1.9) tells us that there are indeed 24 plane locations, and because we have only 18 independent parameters, the locations of the 7-branes cannot be all independent, but must be to some degree correlated. Moreover, the heterotic compactification has $16+2=18 U(1)$ factors (besides the graviphotons), while we have on the $F$-theory side 24 7-branes, each of which carries locally a $U(1)$ factor. Therefore the degrees of freedom of the 24 branes must somehow be restricted globally, though locally all the branes look the same.

The point is that there are in fact many types of 7-branes, labelled by their electric and magnetic charges $(p, q)$. A $D$ 7-brane is by definition a brane on which a fundamental type IIB string can end, and by convention carries $(p, q)$ charge of $(0,1)$. Conversely, a 7-brane on which a $D 1$ string can end has $(p, q)=(1,0)$, and the generic $(p, q)$ brane is a 7 -brane on which a $(p, q)$ string [15] can end.

The $S L(2, \mathbb{Z})$ S-duality group acts on the charge labels ${ }^{t}(p, q)$ in the obvious manner, and all the $S L(2, \mathbb{Z})$ orbits of a given brane have locally identical properties. Indeed the monodromy induced by encircling a $(p, q)$ brane is simply the corresponding $S L(2, \mathbb{Z})$ conjugate of the monodromy $\mathcal{M}_{(0,1)}=\left(\begin{array}{ll}1 & 1 \\ 0 & 1\end{array}\right)$ of a single $D 7$-brane, and takes the following form:

$$
\mathcal{M}_{(p, q)}=\left(\begin{array}{cc}
1+p q & q^{2} \\
-p^{2} & 1-p q
\end{array}\right) \in S L(2, \mathbb{Z}) .
$$

Global consistency then requires that the total monodromy on the $\mathbb{P}^{1}$ base must be trivial:

$$
\prod_{i=1}^{24} \mathcal{M}_{\left(p_{i}, q_{i}\right)}=\mathbf{1}
$$

which obviously forbids all 24 branes to simultaneously be of type $(0,1)$.

A given pair of 7-branes is said to be "mutually local" if their monodromies commute, which is when

$$
p_{1} q_{2}-p_{2} q_{1}=k \in \mathbb{Z}
$$

vanishes. Then they can be simultaneously treated at weak coupling, using methods of ordinary conformal field theory. If on the other hand the intersection number $k \neq 0$, then the branes are mutually "nonlocal" or "dyonic", and cannot simultaneously be described at weak

\footnotetext{
${ }^{4}$ Mathematically, this label refers to the homology class of the vanishing 1-cycle $\gamma$ that characterizes the singular elliptic fiber $T^{2}$. That is, $\gamma=p \alpha+q \beta$, where $\alpha, \beta$ form a symplectic basis of $H_{1}\left(T^{2}, \mathbb{Z}\right)$.
} 
coupling. In particular, the naive addition of the $U(1)$ gauge groups that locally live on each brane, is then ill-defined. Since the global consistency condition (1.12) turns out to admit only a maximum of 18 mutually commuting monodromies, it is thus clear that we cannot expect to see more than 18 independent $U(1)$ factors in the full theory, even though each of the 24 branes carries one of such factors locally. Therefore there is generically no weak coupling, or CFT description description that would be valid for the complete system of 24 branes.

\subsection{GAUGE SYMMETRIES AND KODAIRA SINGULARITIES}

We have mentioned in the preceding section that the moduli space $\mathcal{M}$ (1.10) of elliptic K3's is the same as of the heterotic string compactified on $T^{2}$; at generic points in $\mathcal{M}$ the gauge symmetry is simply $U(1)^{18}$. However, there is much more structure in the theory, in particular at specific sub-loci of $\mathcal{M}$, extra non-abelian gauge symmetries can appear. This is familiar for the heterotic string, where for example we can switch off the Wilson lines and thereby restore the $E_{8} \times E_{8}$ gauge symmetry of the ten dimensional theory. In fact there can appear any combination of "simply laced" gauge groups (those which are classified by the labels $A_{n}, D_{n}$ and $\left.E_{6,7,8}\right)$, as long as the total rank does not exceed 18.

An immediate question is therefore how such gauge symmetries appear in the $F$-theory language. For the $A_{n} \sim S U(n+1)$ type of groups, the answer is well-known and simple: if we place $n D 7$-planes near each other, then the open strings that are stretched in all possible ways between them describe massive charged gauge bosons of $U(n) / U(1)^{n}-$ see Fig. 1.3 a). If the branes collide, these $n(n-1)$ strings will have zero masses and so lead to a gauge enhancement, $U(1)^{n} \rightarrow U(n)$.

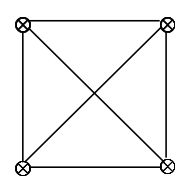

a)

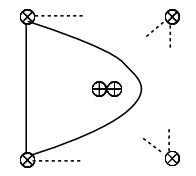

b)

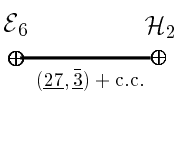

c)

Figure 1.3 Enlargement of non-abelian gauge symmetries: a) $U(n)$ is generated by open strings stretched between $n$ D7-branes of type $(0,1) ; b)$ if we add a pair of $(1,1)$ and $(1,-1)$ branes, then the extra "indirect" trajectories extend $U(n)$ to $S O(2 n)$; c) $E_{8}$ is generated by colliding $\mathcal{E}_{6}$ and $\mathcal{H}_{2}$ Kodaira 7-planes.

For the other gauge groups the story is not that simple, however. To understand it, let us rephrase what we just said in terms of $K 3$ geometry. Here each D7-brane corresponds to a singular elliptic fiber 
with type $(0,1)$ vanishing 1 -cycle, located over the same point in the $z$ plane. Accordingly, the gauge enhancement corresponds in this language to the collision of singular fibers. In particular, when all singular fibers are on top of each other, then the resulting singular fiber will have a worse singularity than we had before (see Fig. 1.4).

Actually, upon collision not only the elliptic fiber, but also total space, which is the $K 3$ surface, becomes singular or more singular. To see this, note that the open string stretched between any $D 7$-branes is nothing but a projection of a 2-cycle in the $K 3$ - see again Fig. 1.4. This 2-cycle can be visualized by dragging the $(0,1)$ cycle in the fiber along the path of the open string (in other words, the 2-cycle is a fibration of $S^{1}$ over a line segment). If the $D 7$-branes collide, then the 2 -cycle obviously shrinks to zero size, and this is precisely what makes the $K 3$ singular.

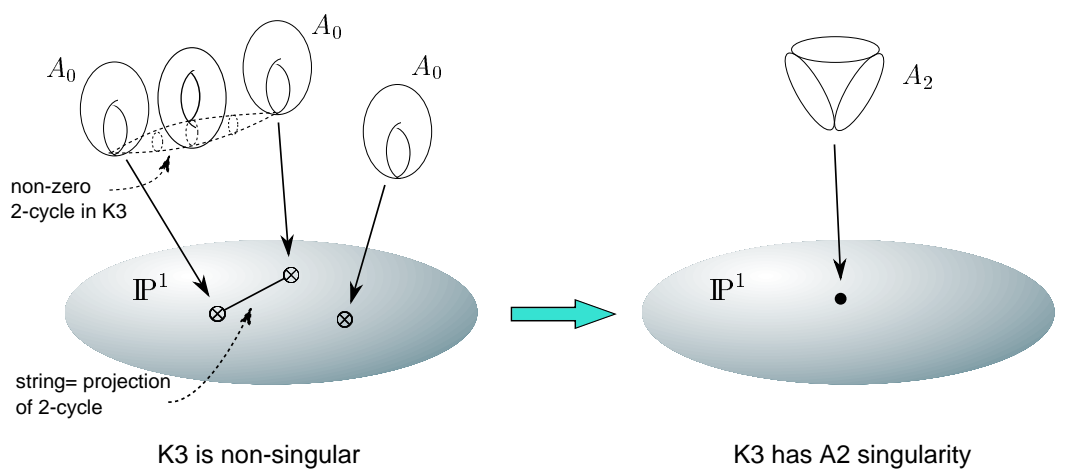

Figure 1.4 One the left we show three singular fibers of type $A_{0}$. The open string trajectories are projections of 2-cycles of non-zero volume in the $K 3$. On the right we have collided the singular fibers to form a singularity of type $A_{2}$, which is associated with the simultaneous vanishing of several intersecting 2 -cycles in the $K 3$.

There is a mathematical classification [16] of what possible singularities an elliptic $K 3$ surface can have.5 These are essentially given by the well-known ADE singularities, however the condition of being elliptic fibrations gives some little extra structure. The possible elliptic "Kodaira" singularities are listed in Table 1; remember, though, that for a compact $K 3$ surface the total rank that can be achieved is restricted to be less or equal to 18. The precise manner how the open string trajectories build up the various charged gauge bosons can in general be quite complicated, and this is an interesting subject that has been studied

${ }^{5}$ We mean here singularities that can be reached by going a finite distance in moduli space; there can be other kinds of singularities like decompactification limits. 
in detail in various papers [17]; however it is beyond the scope of this review.

\begin{tabular}{llll}
\hline Type & Gauge symmetry & 7 - brane content & Monodromy \\
\hline $\mathcal{A}_{n}$ & $U(n+1)$ & $A^{n+1}$ & $T^{n+1}$ \\
$\mathcal{D}_{n+4}$ & $S O(2 n+8)$ & $A^{n+4} B C$ & $S^{2} T^{-n}$ \\
$\mathcal{E}_{6}$ & $E_{6}$ & $A^{5} B C^{2}$ & $(S T)^{2}$ \\
$\mathcal{E}_{7}$ & $E_{7}$ & $A^{6} B C^{2}$ & $S$ \\
$\mathcal{E}_{8}$ & $E_{8}$ & $A^{7} B C^{2}$ & $S T$ \\
$\mathcal{H}_{0}$ & $U(1)$ & $A C$ & $(S T)^{-1}$ \\
$\mathcal{H}_{1}$ & $U(2)$ & $A^{2} C$ & $S^{-1}$ \\
$\mathcal{H}_{2}$ & $U(3)$ & $(A C)^{2}$ & $(S T)^{-2}$ \\
\hline
\end{tabular}

Table 1: Kodaira classification of elliptic singularities $(n \geq 0)$. Physically, these correspond to 7-planes which support the respective $A D E$ gauge symmetries on their world-volumina; many of them cannot be described in term of weak coupling physics. We also indicated their (non-unique) decomposition in terms of 7-brane building blocks, where $A$ denotes a 7 -brane of type $(0,1), B$ of type $(1,-1)$ and $C$ of type $(1,1)$. Moreover we show their $S L(2, \mathbb{Z})$ monodromies in terms of the usual generators $S$ and $T$.

Physically, associated to each of these singularities is something what we may call a "Kodaira 7-plane", which may be thought of as superposition or bound state of ordinary 7-branes. We call these object "planes" rather than branes to emphasize that their physical properties are in general quite different from their ordinary brane building blocks. For example, as will be important later on, some of these planes have finite order monodromies, which implies that no logarithmic branch cuts emanate from them. This in turn means that these planes have no net $\mathbb{Z}$-valued $D$-brane charge, and one may view their finite $\mathbb{Z}_{N}$ monodromy as a "torsion" generalization of ordinary $D$-brane charge. Those planes may also be regarded as non-perturbative $\mathbb{Z}_{N}$ generalizations of orientifold planes (which are associated with $\mathbb{Z}_{2}$ ).

An important physical point is furthermore that for the Kodaira singularities other than $\mathcal{A}_{n}$, the vanishing 1-cycles of the colliding singular fibers are not all of type $(0,1)$. This means that all other 7 -planes apart from those of type $\mathcal{A}_{n}$, involve 7 -brane building blocks of different $(p, q)$ types. In fact one can build all the possible 7-planes out of just three kinds of building blocks [17], which are indicated in Table 1 as well.

These building blocks are non-local with respect to each other, and according to our previous considerations, this implies that the 7-planes are generically strongly coupled and cannot be described by ordinary 
CFT methods; it is likely that there isn't any lagrangian description of their world-volumina theories. The exceptions include of course the planes of type $\mathcal{A}_{n}$, and to some extent also $\mathcal{D}_{n+4}$; the latter can be described on a sub-locus of the moduli space where one puts the $(1,1)$ and $(1,-1)$ branes on top of each other; the resulting object behaves like an orientifold plane, which can indeed be dealt with in terms of standard CFT [18].

\subsection{CONSTANT COUPLING SLICES OF THE MODULI SPACE}

For our purpose of computing effective interactions induced by the 7-branes, the mutual non-localities of the $24(p, q)$ branes are very inconvenient. However, what we can do to simplify matters is to restrict to slices of the moduli space where the 24 branes combine into composites that have commuting net monodromies.

Particularly simple are the sub-cases where the monodromies not only commute, but are also of finite order. These correspond to theories where the 7-brane charge is cancelled locally, such that the type IIB string coupling $\tau_{I I B}$ is constant over the $\mathbb{P}^{1}$ base. Remembering from (1.8) that the coupling is determined by

$$
J\left(\tau_{I I B}(z)\right)=\frac{4\left(24 f_{8}(z)\right)^{3}}{4 f_{8}(z)^{3}+27 g_{12}(z)^{2}} \stackrel{!}{\equiv} \text { const. }
$$

we see that requiring $z$-independence yields three (partially overlapping) possibilities for splitting up the 24 branes [18, 19]:

i) $g_{12}=0 \rightarrow J=1728 \rightarrow \tau_{I I B}=i$ : group into eight $\mathcal{H}_{1}$-planes (after fixing three points due to $S L(2, \mathbb{C})$ invariance, this gives five independent moduli)

ii) $f_{8}=0 \rightarrow J=0 \rightarrow \tau_{I I B}=\rho \equiv e^{2 \pi i / 3}$ : group into into twelve $\mathcal{H}_{0}$-planes (nine independent moduli)

iii) $f_{8}=h_{4}{ }^{2}, g_{12}=$ const. $h_{4}{ }^{3} \rightarrow \tau_{I I B}=$ arbitrary constant: group into four $\mathcal{D}_{4}$-planes (one independent modulus; this branch intersects branches ii) and iii)).

Upon further specialization, one can have some or all of the $\mathcal{H}_{n}$ branes combine into planes with larger gauge symmetries; the possibilities are summarized in Table 2. 


\begin{tabular}{|c|ccccccc|}
\hline Kodaira type $\rightarrow$ & $\mathcal{H}_{0}$ & $\mathcal{H}_{1}$ & $\mathcal{H}_{2}$ & $\mathcal{D}_{4}$ & $\mathcal{E}_{6}$ & $\mathcal{E}_{7}$ & $\mathcal{E}_{8}$ \\
\hline Composition & $\mathcal{H}_{0}$ & $\mathcal{H}_{1}$ & $\mathcal{H}_{0}{ }^{2}$ & $\mathcal{H}_{0}{ }^{3}, \mathcal{H}_{1}{ }^{2}$ & $\mathcal{H}_{0}{ }^{4}$ & $\mathcal{H}_{1}{ }^{3}$ & $\mathcal{H}_{0}{ }^{5}$ \\
Torsion Charge & $\mathbb{Z}_{6}$ & $\mathbb{Z}_{4}$ & $\mathbb{Z}_{3}$ & $\mathbb{Z}_{2}$ & $\mathbb{Z}_{3}$ & $\mathbb{Z}_{4}$ & $\mathbb{Z}_{6}$ \\
$\tau_{I I B}$ & $\rho$ & $i$ & $\rho$ & any & $\rho$ & $i$ & $\rho$ \\
\hline
\end{tabular}

Table 2: List of 7-planes with finite order monodromies in the z-plane,

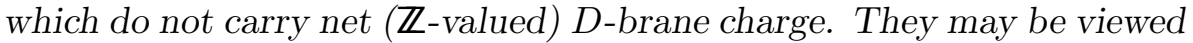
as non-perturbative $\mathbb{Z}_{N}$ generalizations of orientifold planes. We also list their composition in terms of basic $\mathcal{H}_{k}$ building blocks, as well as the associated constant type IIB string coupling, $\tau_{I I B}$.

What we will do, in order to further simplify the problem, is to consider just one-dimensional slices of these moduli spaces, obtained by grouping the $\mathcal{H}_{k}$ planes into four planes in total. As we will see, the corresponding geometries will be then pretty easy to deal with. Explicitly, we will consider from now on the singular $K 3$ surfaces defined by the equations $W(x, y, z ; \lambda)=0$, where:

$$
\begin{array}{ll}
\left(E_{8}^{2} H_{0}^{2}\right): & W=y^{2}+x^{3}+z^{5}(z-1)(z-\lambda) \\
\left(E_{7}{ }^{2} H_{1}^{2}\right): & W=y^{2}+x^{3}+x z^{3}(z-1)(z-\lambda) \\
\left(E_{6}{ }^{2} H_{2}^{2}\right): & W=y^{2}+x^{3}+z^{4}(z-1)^{2}(z-\lambda)^{2} \\
\left(D_{4}^{2} D_{4}^{2}\right): & W=y^{2}+x^{3}+z^{3}(z-1)^{3}(z-\lambda)^{3} .
\end{array}
$$

The first one yields $E_{8} \times E_{8}^{\prime}$ gauge symmetry, and thus will correspond to the heterotic model with switched-off Wilson lines of section 2. The other cases correspond to similar models with certain Wilson lines switched on; we include them here because we can uniformly treat all these models in the same way.

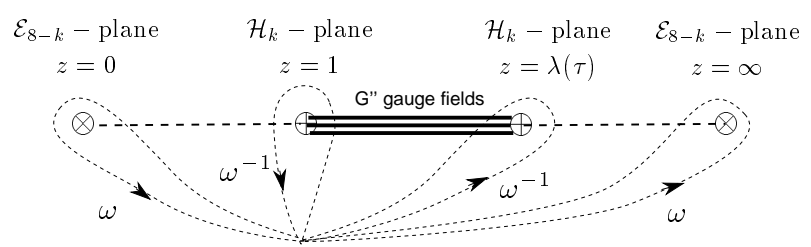

Figure 1.5 7-plane configuration of the first three models in (1.15), which describes $K 3$ surfaces with elliptic $\left(\mathcal{E}_{8-k} \times \mathcal{H}_{k}\right)^{2}$ singularities. We have indicated the monodromies given by $\omega=e^{2 \pi i / N}$, and also exhibited multiplets of (mutually non-local) open strings that run between the $\mathcal{H}_{k}$ planes. For $\lambda(\tau) \rightarrow 1$ the $\mathcal{H}_{k}$ planes merge into a single plane, the strings between them then giving rise to massless charged gauge fields that enhance the non-abelian gauge group.

Each of the surfaces in (1.15) has two pairs of singular fibers of the indicated types over the $z$-plane. As convention we have chosen to 
put 7 -planes of types $\mathcal{E}_{8}, \mathcal{E}_{7}, \mathcal{E}_{6}, \mathcal{D}_{4}$ at $z=0, \infty$, and planes of types $\mathcal{H}_{0}, \mathcal{H}_{1}, \mathcal{H}_{2}, \mathcal{D}_{4}$ at $z=1, \lambda$, respectively (see Fig. 1.5). Note that the Kodaira singularity types of these two sets are "dual" to each other, in that the monodromies of the $\mathcal{E}_{8-k}$ planes and of the $\mathcal{H}_{k}$ planes are inverses of each other; they belong to $\mathbb{Z}_{N}, N=2,3,4,6$, respectively.

In the one-dimensional sub-moduli spaces, two interesting things can happen. First, a $\left(\mathcal{E}_{8-k}\right)$ - and a $\mathcal{H}_{k}$-plane can collide, to yield an " $\overline{\mathcal{E}}_{8}$ " singularity of the local form $y^{2}+x^{3}+z^{6}=0$. As we will see, this corresponds to the decompactification limit on the heterotic side. Secondly, two $\mathcal{H}_{k}$-planes can collide to produce a 7 -plane associated with some extra non-abelian gauge symmetry $G^{\prime \prime}$, and precisely which one can be inferred from Table 2. In other words, the generic non-abelian gauge symmetry is $\left(E_{8-k} \times A_{k}\right)^{2}$, which can be enhanced to $\left(E_{8-k}\right)^{2} \times G^{\prime \prime}$, for $G^{\prime \prime}=A_{2}, D_{4}, E_{6}$, respectively (for colliding $\mathcal{D}_{4}$ planes there is no further gauge enhancement, as this also corresponds to the decompactification limit).

\section{GEOMETRIC DETERMINATION OF THE THRESHOLD COUPLINGS \\ 4.1 CHERN-SIMONS COUPLINGS ON KODAIRA 7-PLANES}

The issue is to compute the functions $\Delta_{\text {eff }}(1.1)$ via 7 -brane interactions. Effective interactions in $8 d$ space-time are generated by superimposing world-volume actions, and also by integrating out exchanges between the 7-branes. While in general very complicated, the interactions are in the present context reasonably tractable because of their special anomaly related, parity-odd structure. They arise from the ChernSimons terms on the world-volumina of the 7-branes, via the exchange of $R R$ antisymmetric tensor fields $C^{(p)}$.

For a single $D$-brane with $(p, q)=(0,1)$, the relevant tree level couplings look [20] (for trivial normal bundle):

$$
\mathcal{L}_{D 7}^{(\mathrm{CS})}=\left.C \wedge e^{-2 i F} \wedge \sqrt{\widehat{\mathcal{A}}(R)}\right|_{8-\text { form }}
$$

where $C \equiv \oplus_{k=0}^{4} C^{(2 k)}$ is the formal sum over all $R R$ forms, and $\widehat{\mathcal{A}}(R)$ is the Dirac genus. The couplings for general $(p, q)$ branes can be obtained by applying $S L(2, \mathbb{Z})$ transformations on the fields in (1.16).

However, due to the generic mutual non-locality of the $24(p, q) 7$ branes that we simultaneously have in the theory, it is a priori not clear how to add up these terms and how to determine what effective interactions they induce. But as discussed above, we can simply restrict 
to sub-moduli spaces where the 24 branes combine into 7 -planes that are all mutually local, ie., have commuting monodromies. Then all the contributions can simply be added up.

In order to do so, we will first need to know what the relevant couplings on the world-volumina on the various kinds of 7-planes are, in analogy to the couplings on a single D7-brane (1.16). Because of the mutual locality, the anomalous couplings can in fact be very easily determined. Specifically, recall that a $\mathcal{D}_{4}$-plane can be viewed as being composed out of four D7-branes plus one orientifold plane, which are all mutually local. Since a direct CFT computation gives $\mathcal{L}_{O 7}^{(\mathrm{CS})}=-\left.4 C \wedge \sqrt{\widehat{\mathcal{L}}(R)}\right|_{8 \text {-form }}$ $[21,22,23]$, where $\widehat{\mathcal{L}}(R)$ is the Hirzebruch genus, we thus have:

$$
\begin{aligned}
\left.\mathcal{L}_{\mathcal{D}_{4}}^{(\mathrm{CS})=} \quad C \wedge\left[\operatorname{tr}\left(e^{-2 i F}\right) \wedge \sqrt{\widehat{\mathcal{A}}(R)}-4 \sqrt{\widehat{\mathcal{L}}(R)}\right]\right|_{8-\text { form }} \\
=C^{(4)} \wedge\left(\frac{1}{2} R^{2}-2 \operatorname{tr} F^{2}\right) \\
\quad+C^{(0)} \wedge\left(\frac{2}{3} \operatorname{tr} F^{4}-\frac{1}{12} \operatorname{tr} F^{2} \operatorname{tr} R^{2}+\frac{1}{192}\left(\operatorname{tr} R^{2}\right)^{2}+\frac{1}{48} \operatorname{tr} R^{4}\right)
\end{aligned}
$$

Summing over all four world-volumina indeed exactly reproduces the (eight dimensional remainder of the) Green-Schwarz term of the heterotic string, $\mathcal{L}^{(G S)}=C^{(6)} \wedge 2\left(R^{2}-\operatorname{tr} F_{S O(32)}{ }^{2}\right)+C^{(2)} \wedge X_{8}\left(F_{S O(32)}, R\right)$.

The same logic must be valid for the $\mathcal{H}_{0}$ and $\mathcal{H}_{1}$-planes and their composites. Even though these planes are associated with strong coupling and may not have a well-defined lagrangian description of their world-volume theories, the WZ coupling terms are topological and independent of the coupling, and must make sense at least for anomaly

cancelling reasons. Therefore, we can conclude for the basic building blocks:

$$
\mathcal{L}_{\mathcal{H}_{0}}^{(\mathrm{CS})}=\frac{1}{3} \mathcal{L}_{\mathcal{D}_{4}}^{(\mathrm{CS})}, \quad \mathcal{L}_{\mathcal{H}_{1}}^{(\mathrm{CS})}=\frac{1}{2} \mathcal{L}_{\mathcal{D}_{4}}^{(\mathrm{CS})},
$$

where the gauge field traces follow implicitly from the decomposition $S O(8) \rightarrow U(1)$ or $U(2)$, respectively.

\subsection{GEOMETRIC INTERACTIONS ON $\mathbb{Z}_{N}$ CURVES}

What we are interested in are the non-trivial interactions between the planes, which should ultimately reproduce the coupling functions $\Delta_{\text {eff }}(T, U=$ const) of section 2 . The primary perturbative contributions will arise from massless $C^{(p)}$ tensor field exchange between individual planes. The effective interaction will thus depend on the distances between the various 7 -planes in the $z$-plane. 
More specifically, the closed string exchange that contributes to the maximal number of wedge products of field strengths is in the odd $R R$ sector, and is proportional to the Green's function $\Delta$ of a scalar field on the $z$-plane:

$$
\left\langle C_{m_{1} \ldots m_{p}}^{(p)}\left(z_{1}\right), C_{n_{1} \ldots n_{8-p}}^{(8-p)}\left(z_{2}\right)\right\rangle_{R R^{-}} \sim \epsilon^{m_{1} \ldots m_{p} n_{1} \ldots n_{8-p}} \Delta\left(z_{1}, z_{2}\right)
$$

where, as $z_{1} \rightarrow z_{2}$ :

$$
\Delta\left(z_{1}, z_{2}\right)=\ln \left(z_{1}-z_{2}\right)+\text { finite }
$$

However, in order to obtain functionally exact results, we need to know the full Green's functions that probe the global structure of the $z$-plane, and not just their leading singular behavior. This is in general a complicated problem, but in our setup, where we consider only planes with finite order monodromies, there is a natural geometric answer $[5,6]$.

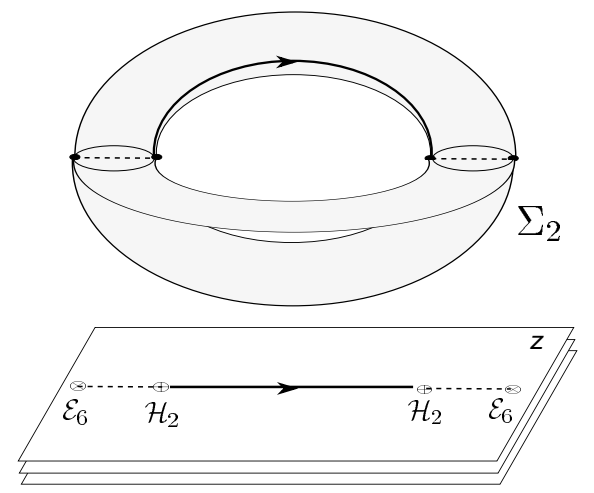

Figure 1.6 Lift of the $z$-plane to a covering Riemann surface. Shown is here the situation with two $\mathcal{E}_{6}$ and two $\mathcal{H}_{2}$ planes, which correspond to $\mathbb{Z}_{3}$ twist fields and anti-twist fields, respectively, located at the branch points of a genus two curve $\Sigma_{2}$. We also show an open string trajectory that contributes to the coupling $\operatorname{tr} F_{S U(3)}{ }^{2} \operatorname{tr} F_{S U(3)^{\prime}}{ }^{2}$ (transforming as $(3, \overline{3})$ under $S U(3) \times S U(3)^{\prime} \subset E_{6}$ ) and which corresponds to a $1 / 3-$ period on $\Sigma_{2}$.

By definition, monodromies of finite order means that the geometry of the singular $K 3$ 's can be described by a finite covering of the $z$-plane and so effectively reduces to the one of Riemann surfaces. The four 7planes then correspond to the branch points of these curves (cf. Fig. 1.6). More specifically, for the four models in (1.15) one finds the following $\mathbb{Z}_{N \text {-symmetric curves }}$

$$
\Sigma_{N-1}: \quad x^{N}=z^{-1}(z-1)(z-\lambda)
$$

of genus $g=N-1$, where $N=6,4,3,2$, respectively. The requisite Green's functions should therefore simply be given by appropriate scalar Green's functions on these covering spaces [24]. 
The canonical Green's function of a scalar field on a Riemann surface is known to be given by the logarithm of the "prime form"

$\Delta_{\substack{\text { prime } \\ \text { form }}}^{\Sigma}\left(z_{1}, z_{2}\right)=\ln \left|\frac{\theta_{\delta}\left[\int_{z_{1}}^{z_{2}} \vec{w} \mid \Omega\right]}{\sqrt{\xi\left(z_{1}\right)} \sqrt{\xi\left(z_{2}\right)}}\right|-\pi\left[\operatorname{Im} \int_{z_{1}}^{z_{2}} \vec{w}\right] \cdot(\operatorname{Im} \Omega)^{-1} \cdot\left[\operatorname{Im} \int_{z_{1}}^{z_{2}} \vec{w}\right]$,

where $\sqrt{\xi(z)} \equiv \sqrt{\frac{\partial}{\partial z_{i}}\left(\theta_{\delta}[\vec{z} \mid \Omega]\right) \cdot w^{i}(z)}$ is a some $1 / 2$-differential whose purpose is to cancel spurious zeros of the numerator theta-function (where $\delta$ denotes an arbitrary odd characteristic). Indeed, the only singularity of the prime form is at coincident points, ie., $\Delta^{\Sigma}\left(z_{1} \rightarrow z_{2}\right) \sim$ $\ln \left[\frac{z_{2}-z_{1}}{\sqrt{d z_{1}} \sqrt{d z_{2}}}\right]+$ finite terms. By construction, the finite terms implement the requisite global properties of the Green's functions.

Due to the high degree of symmetry of our $\mathbb{Z}_{N}$ curves $\Sigma_{N-1}$, much of the information in (1.22) is in fact redundant for our examples. By explicit computation one can show that the prime form Green's function, when evaluated between any two of the branch points, can always be written in the following generic form:

$$
\underset{\substack{\text { prime } \\ \text { form }}}{\Sigma_{N-1}}\left(z_{1}, z_{2}\right)=\ln \left[\lambda^{\alpha_{1}}(1-\lambda)^{\alpha_{2}}\left(\lambda^{\prime}\right)^{3}\right],
$$

where $z_{1,2} \in\{0,1, \lambda, \infty\}, z_{1} \neq z_{2}$ and the numerical coefficients $\alpha_{i}$ depend on the particular choice of $z_{1}$ and $z_{2}$.

However, it turns out [6] that these canonical Green's functions on $\Sigma_{N-1}$ to not capture the full story. They capture only the exchange of $C$ fields, but miss certain additional instanton contributions. Namely, loops of $(p, q)$ strings in the $z$-plane will be closed in general only on the covering surface $\Sigma_{N-1}$, so that such strings effectively wrap the Riemann surfaces. Wrapping entire world-sheets of such strings will thus in general generate extra instanton contributions

These extra contributions can be viewed as modifications of the canonical Green's functions (1.23) into "effective" Green's functions. Indeed, a Green's function is in general ambiguous up to the addition of a finite piece, and it is this ambiguous piece to which we can formally attribute those extra non-singular, non-perturbative corrections. Denoting the extra piece by $\delta$, we can thus write the threshold coupling functions generically as follows:

$$
\Delta_{\text {eff }}(\lambda)=\Delta_{\substack{\text { prime } \\ \text { form }}}^{\Sigma_{N-1}}(\lambda)+\delta(\lambda)
$$

We will describe further below how to exactly compute the extra contributions $\delta(\lambda)$.

For the time being, note that the above picture applies most directly to couplings that mix the gauge field strengths of two different 7 -planes, 
which means that they have the form $\left\langle C^{(4)}, C^{(4)}\right\rangle \times \operatorname{tr} F_{G}^{2} \wedge \operatorname{tr}{F_{G^{\prime}}}^{2}$. However, as one can check on the heterotic side, there are moduli-dependent corrections also to other eight-form terms in the effective action, eg., to $\left(\operatorname{tr} F_{G}^{2}\right)^{2}$, which pertain to a single gauge group factor living on a single brane.

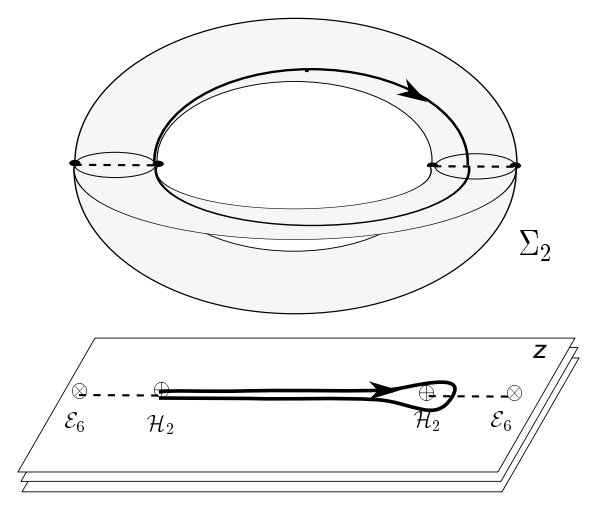

Figure 1.7 Interactions probing the $\mathbb{Z}_{3}$ torsion piece of $D$-brane charge. A string junction is shown that contributes to $\left(\operatorname{tr} F_{S U(3)}{ }^{2}\right)^{2}$, transforming as a singlet under $S U(3) \times S U(3)^{\prime} \subset E_{6}$. We also show how it lifts to a cycle on the covering curve. The junction gives rise to a logarithmic singularity when the planes collide, even though it does not seem to couple locally to the right $\mathcal{H}_{2}$ plane.

In the usually considered situation with CFT description, where one focuses on pairs of $D$ - or orientifold-branes [21, 22, 23], such terms arise from integrating out $C^{(0)}-C^{(8)}$ exchange between the two branes, each equipped with couplings like $\mathcal{L}^{(\mathrm{CS})}=Q_{7} \cdot C^{(8)}+\ldots+C^{(0)} \wedge Y_{8}(F)$, where $Y_{8}(F)$ is some 8-form polynomial. This obviously induces a locationdependent correction to the quartic gauge field coupling of the form $Q_{7}\left\langle C^{(0)}, C^{(8)}\right\rangle \operatorname{tr} Y_{8}\left(F_{G}\right)$.

However, in the present context, the 7-brane charge is cancelled locally on every plane so that $Q_{7} \equiv 0$; indeed there is no $C^{(8)}$ term in (1.17). This means that naive $C^{(0)}-C^{(8)}$ exchange cannot contribute to these couplings. But how do these (possibly singular) moduli-dependent corrections, which we explicitly see on the heterotic side, then arise ? More specifically, how can a given brane that carries the field strength $F_{G}$ "feel" the presence of the other brane, while no string ends on that other brane?

The point is that despite our 7-planes do not have net $\mathbb{Z}$-valued 7 brane charge (no logarithmic monodromy), there is still a remnant left, which is reflected by the finite order $\mathbb{Z}_{N}$ monodromies. It is this "torsion" piece of the $D$-brane charge that must be responsible for the req- 
uisite long-range interactions [25]. This can be seen by analyzing the interactions in terms of string junctions [17]. Similar to what is familiar from orientifold planes, what one finds are string trajectories that loop around other planes, rather than coupling to them via the local ChernSimons terms in (1.17); this is exemplified in Fig. 1.7 for the $E_{6}$ model. It thus seems natural to view these interactions as analogous to those of "Alice strings" [26], which do have long-range interactions but no locally defined charge density.

At any rate, what we learn by studying these couplings is that there can be non-trivial interactions between 7 -branes that should be attributed the global properties of the multi-valued $z$-plane (which is best represented by the curves $\Sigma_{N-1}$ ), rather than solely to local WZ couplings.

\section{SOLUTION VIA THE MIRROR MAP 5.1 FLAT COORDINATES AND MODULI SPACES}

The singular $K 3$ surfaces in (1.15) all depend on one geometric modulus, $\lambda$. On the other hand, the heterotic models we consider depend on two moduli $T, U$ (besides the Wilson lines that we keep frozen and so neglect). In order to compare the geometrical interactions, which depend on the parameter $\lambda$ in (1.23), with the heterotic one-loop results, we therefore -first of all- need to know what the map between these moduli is.

The point is that the heterotic moduli are the canonical moduli of a conformal field theory. From general reasoning [27, 28] we know that the moduli space has a flat structure and that the canonical CFT moduli are the corresponding flat coordinates. Therefore, we need to determine what the flat coordinate $\tau$ is that is associated with the geometric mod-

ulus $\lambda$. A general way of constructing the flat coordinate is to write $\tau(\lambda)$ in terms of the ratio of certain period integrals (which are themselves solutions of certain linear differential equations, as we will discuss below).

Specifically, the relevant periods pertaining to the singular $K 3$ surfaces $W(x, y, z ; \lambda)=0$ in (1.15) are obtained by integrating the unique holomorphic two-form $\Omega_{K 3}^{(2,0)}$,

$$
\varpi_{i}=\int_{\gamma_{i}} \Omega_{K 3}^{(2,0)} \equiv \int_{\gamma_{i}} \frac{d x d z}{\partial_{y} W(x, y, z ; \lambda)}
$$

over a suitable integral basis of 2-cycles $\gamma_{i}$. We have seen before that these singular $K 3$ 's are closely related to Riemann surfaces $\Sigma_{N-1}$, and indeed by redefining variables: $x=v z^{2(1-1 / N)}(z-1)^{2 / N}(z-\lambda)^{2 / N}$, 
the period integrals (1.25) factorize into $\int \frac{d v}{\sqrt{v^{3}+1}} \int \frac{d z}{z^{1-1 / N}(z-1)^{1 / N}(z-\lambda)^{1 / N}}$. The integral over $v$ being a constant normalization that we neglect, the $K 3$ period integrals thus turn into period integrals (now over 1-cycles) pertaining to the $\mathbb{Z}_{N}$ curves (1.21):

$$
\varpi_{i}=\int \frac{d z}{z^{1-1 / N}(z-1)^{1 / N}(z-\lambda)^{1 / N}} \equiv \int_{\gamma_{i}} \Omega_{\Sigma_{N-1}}^{(1,0)}
$$

These integrals are of canonical hypergeometric type and so given by linear combinations of

$$
\begin{aligned}
& \varpi_{0}(\lambda)=\int_{0}^{\lambda} \Omega_{\Sigma_{N-1}}^{(1,0)}=(-1)^{-2 / N} \pi \csc \left(\frac{\pi}{N}\right)_{2} F_{1}\left(\frac{1}{N}, \frac{1}{N}, 1 ; \lambda\right) \\
& \varpi_{1}(\lambda)=\int_{0}^{1} \Omega_{\Sigma_{N-1}}^{(1,0)}=\lambda^{-1 / N}(-1)^{-2 / N} \pi \csc \left(\frac{\pi}{N}\right)_{2} F_{1}\left(\frac{1}{N}, \frac{1}{N}, 1 ; \frac{1}{\lambda}\right) .
\end{aligned}
$$

The flat coordinate is then given, as usual, by the ratio of the periods:

$$
\begin{aligned}
\tau(\lambda) & =\frac{\varpi_{1}(\lambda)}{\varpi_{0}(\lambda)} \\
& =s\left(0,0,1-\frac{2}{N} ; \lambda\right) .
\end{aligned}
$$

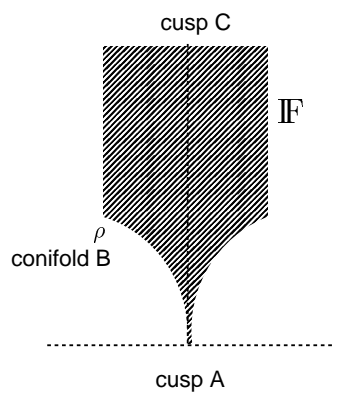

$$
\tau \longleftrightarrow \lambda(\tau)
$$

$\mathrm{F}$

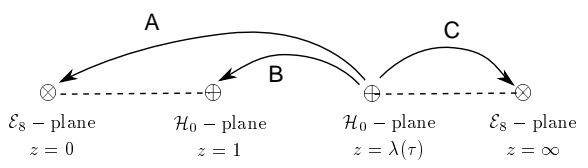

Figure 1.8 On the left we see a picture of the moduli space of the heterotic compactification with $E_{8} \times E_{8}$ gauge symmetry, given by a fundamental region $\mathbb{F}=\mathbb{F}_{\Gamma} \cup\left(S \cdot \mathbb{F}_{\Gamma}\right) \subset \mathbb{H}^{+}$. The Hauptmodul $\lambda(\tau)$ maps this region to the 7 -plane geometry shown in the right part of the figure. The cusps and orbifold point correspond to the various ways the mobile $\mathcal{H}_{0}$ plane can hit the three other planes.

In (1.28), $s(a, b, c)$ denotes a triangle function that maps the complex plane into a fundamental region $\mathbb{F} \subset \mathbb{H}^{+}$, while its entries $a, b, c$ indicate the angles of $\mathbb{F}$. We depict a typical such fundamental region in Fig. 1.8. The two zero's mean that there are generically two cusps (corresponding 
to the decompactification limits $\lambda \rightarrow 0, \infty)$, and in addition there is one orbifold point associated with gauge enhancement, $\lambda \rightarrow 1$ (however, for $N=2$ there are three cusps, which reflects that any two colliding $\mathcal{D}_{4}$ singularities correspond to decompactification).

By the theory of triangle functions, the inverse maps given by the "Hauptmoduls" $\lambda(\tau)$, can then be concisely written in terms of standard modular functions. These, together with the modular subgroups under which $\lambda$ is invariant, are listed in Table 3 .

\begin{tabular}{rlll}
\hline$N$ & mod. subgroup & Hauptmodul $\lambda(\tau=T)$ & $\tau_{I I B}=U$ \\
\hline 6 & $\Gamma_{P}(2)$ & $(\sqrt{-J(\tau)}+\sqrt{1-J(\tau)})^{-2}$ & $\rho$ \\
4 & $\Gamma_{0}(2)$ & $-\frac{1}{64}\left(\frac{\eta(\tau)}{\eta(2 \tau)}\right)^{24}$ & $i$ \\
3 & $\Gamma_{0}(3)$ & $-\frac{1}{27}\left(\frac{\eta(\tau)}{\eta(3 \tau)}\right)^{12}$ & $\rho$ \\
2 & $\Gamma_{0}(4)$ & $-\frac{1}{16}\left(\frac{\eta(\tau)}{\eta(4 \tau)}\right)^{8}$ & undet. \\
\hline
\end{tabular}

Table 3: Modular subgroups of which $\lambda(\tau)$ is a modular function for the $K 3$ families in (1.15), and the values of the constant string coupling $\tau_{I I B}$. They agree with the duality groups of the corresponding dual heterotic string compactifications. (The modular subgroup $\Gamma_{P}(2) \subset S L(2, \mathbb{Z})$ has been discussed in ref. [29]).

If we now identify

$$
\begin{aligned}
T & =\tau \\
U & =\tau_{I I B} \equiv \text { const. }
\end{aligned}
$$

(where the constant type IIB coupling $\tau_{I I B}$ is as listed in Table 3 ), then the above modular subgroups indeed exactly match the modular subgroups that arise on the heterotic side by switching on the corresponding Wilson lines.6

Moreover we can use the map $\lambda(T)$ to compare the leading singularities in the geometric Green's function (1.24) with the heterotic one-loop results. Note that (1.23) carries the leading logarithmic behavior of the full Green's function when two of the $\mathcal{H}_{n}$ planes collide for $\lambda \rightarrow 1$. In particular, for the $E_{8}$ model we get:

$$
\Delta_{\text {eff }}(T) \stackrel{\lambda \rightarrow 1}{\sim} \ln [\lambda(T)] \sim \ln [J(T)],
$$

which captures precisely the singularity of the coupling $\Delta_{\text {eff }}^{(T T U U)}(T, \rho)$ in (1.5) (where we need to set $J(U) \rightarrow J(\rho) \equiv 0$ ). One can check

${ }^{6}$ The functional map between $T, U$ (with $U$ not being frozen) and the $z$-plane geometry has been determined for the $E_{8} \times E_{8}$ model in refs. [30, 5]. 
that similarly for the other models, there is perfect agreement with the singularities in the perturbative results on the heterotic side. This yields a first quantitative, though still superficial test of the duality.

\subsection{STRING GEODESICS AND BPS MASSES}

Remember that the elliptic $K 3$ manifold that appears in $F$-theory is primarily an elegant tool to encode the relevant open string geometry of non-perturbative type IIB compactifications, and as such should have no particular physical significance in itself (unless the fictitious twelve dimensional $F$ theory turns out to exist). Therefore, we should be able to give the geometrical quantities that we have discussed above a physical meaning that makes sense more directly in the type IIB compactification on $\mathbb{P}^{1}$.

Indeed the periods and flat coordinates have a direct interpretation in terms of open strings. Recall [15] that the tension of a $(p, q)$ string (in the canonical metric) is

$$
T_{p, q}=\frac{1}{\sqrt{\operatorname{Im} \tau_{I I B}}}\left|p+q \tau_{I I B}\right|
$$

so that the mass of a string stretched along a line $\mathcal{C}$ is $\int_{\mathcal{C}} T_{p, q} d s$. The line element has been determined in $[31,18]$ and is given by $d s^{2}=$ $\left.\operatorname{Im} \tau_{I I B}\left|\eta\left(\tau_{I I B}\right)\right|^{2} \prod_{i=1}^{24}\left(z-z_{i}\right)^{-1 / 12} d z\right|^{2}$. For the geometries we consider (with four 7-planes and constant type IIB coupling $\tau_{I I B}$ ), the mass of a string stretched between any two planes (at branch points $z_{1}$ and $z_{2}$ ) then simplifies to:

$$
\begin{aligned}
m_{p, q}{ }^{2} & =\int_{z_{1}}^{z_{2}}|\underbrace{\left(p+q \tau_{I I B}(z)\right) \eta^{2}\left(\tau_{I I B}(z)\right)}_{\text {const. }} z^{1 / N-1}(z-1)^{-1 / N}(z-\lambda)^{-1 / N} d z|^{2} \\
& =\left|p+q \tau_{I I B}\right|^{2}\left|\eta^{2}\left(\tau_{I I B}\right)\right|^{2} \int_{z_{1}}^{z_{2}}\left|\Omega_{\Sigma_{N-1}}^{(1,0)}\right|^{2}
\end{aligned}
$$

which coincides up to a numerical prefactor with the period integral (1.26) (strictly speaking, period integrals are over closed homology cycles, while here the open strings are stretched along half-cycles. The difference is just in the normalization). In fact only ratios of periods have an invariant meaning, whence we have to divide (1.31) by the fundamental period $\varpi_{0}$. To see that this provides the correct normalization, consider in the $E_{8} \times E_{8}$ model the mass of open strings stretched between the two $\mathcal{H}_{0}$ planes. It is proportional to

$$
m_{p, q}{ }^{2} \sim\left|p+q \tau_{I I B}\right|^{2}\left|\frac{\int_{1}^{\lambda} \Omega_{\Sigma_{5}}^{(1,0)}}{\int_{0}^{\lambda} \Omega_{\Sigma_{5}}^{(1,0)}}\right|^{2}
$$




$$
=|p+q \rho|^{2}\left|\frac{\tau-\rho}{\tau-\bar{\rho}}\right|^{2} .
$$

With the identification (1.29): $\tau_{I I B} \equiv \rho, \tau=T$, this exactly reproduces the known heterotic mass formula for the $S U(3) / U(1)^{2}$ gauge bosons that become massless as the planes collide (as $\lambda \rightarrow 1$ or $T \rightarrow \rho$ ). The open strings that correspond to these gauge bosons are sketched in Figs. 1.5 and 1.6; they have $(p, q)$ charges given by $\pm(1,0), \pm(0,1)$ and $\pm(1,-1)$, respectively, for which (1.32) implies that they all have the same mass.

One can check that the BPS masses of stretched strings match also in the other examples those of the corresponding winding and momentum states in the heterotic string compactifications on $T^{2}$; a detailed analysis has been presented in ref. [32].

\subsection{MIRROR MAP ACTING BETWEEN OPEN AND CLOSED STRING SECTORS}

Note that in the previous section, we tacitly used a different language as before: namely we used the concept of stretched open strings, while previously we had discussed $C$-field exchange between 7 -planes, which is primarily a closed string concept. Indeed the mass of stretched open strings is best parametrized by the flat coordinate $T$, on the other hand closed string interactions between 7-planes depend on their physical locations in the $z$-plane, and thus are more naturally parametrized by the geometrical modulus $\lambda(T)$.

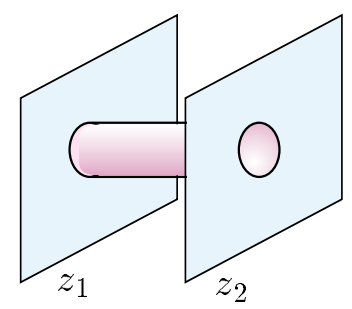

Figure 1.9 Dual interpretations of the same string diagram, obtained by time-slicing the world-sheet in two orthogonal ways: either in terms of tree level closed string exchange between the planes, leading to a contribution $<C, C>\sim \ln \left[z_{1}-z_{2}\right]$. Or as one-loop diagram involving stretched open strings, leading to $\sum Q_{i}{ }^{4} \ln \left[m_{i}\right]$. The functional relationship between these expressions is essentially governed by the mirror map, $\tau \leftrightarrow \lambda(\tau)$.

That a given physical string process can have different interpretations in terms of open or closed strings is of course a well-known, basic fact of string perturbation theory - see Fig. 1.9. To see this better in the present 
context, let us recapitulate the origin of the logarithmic singularity that arises in the effective action when two $\mathcal{H}_{0}$ planes collide in the $E_{8} \times E_{8}$ model. It can be seen to arise in following two dual ways:

i) It either arises as leading one-loop effect involving open strings stretched between the planes:

$$
\Delta_{\text {eff }} \sim \sum_{\substack{\text { states } \\ i}} Q_{i}{ }^{4} \ln \left[m_{i}\right]
$$

which gives, using (1.32):

$$
\Delta_{\text {eff }} \sim 3 \ln \left[\frac{T-\rho}{T-\bar{\rho}}\right] .
$$

The numerical factor arises due to the 3 sets of charged $S U(3) / U(1)^{2}$ gauge bosons which have the same mass.

ii) Alternatively, the singularity arises -as discussed before- from massless closed string $C$-field exchange between the planes, namely as smalldistance singularity of a Green's function:

$$
\Delta_{\text {eff }} \sim\left\langle C^{(4)} C^{(4)}\right\rangle \sim \ln \left[z_{1}-z_{2}\right] \sim \ln [1-\lambda(T)] \sim \ln [J(T)] .
$$

The $J$-function then expands near the singularity as

$$
J(T \simeq \rho)=\text { const. }\left(\frac{T-\rho}{T-\bar{\rho}}\right)^{3}+\ldots,
$$

where the power indeed reproduces the same prefactor as in (1.34). Thus the $J$-function (which pertains to the geometry of the $z$-plane) "knows" about the open string states of the theory, and effectively sums up many contributions in a duality invariant way.

The insight that we can abstract from this is that the map $\tau \leftrightarrow$ $\lambda$ between flat and geometric coordinates of the moduli space can be physically interpreted in terms of a map between the natural open and closed string moduli. From the view point of $K 3$ period integrals, the map $\tau \leftrightarrow \lambda$ can also be viewed as mirror map [33] on K3] However, in the $F$-theory setup the whole of $K 3$ is not really physical, and we should not consider closed two-cycles but rather their projection on the $z$-plane, which gives open string geodesics. In this sense, the rôle of the $K 3$ mirror map in the physical type IIB compactification on $\mathbb{P}^{1}$ is then played by a map between open and closed string sectors.

\footnotetext{
${ }^{7}$ While for higher dimensional Calabi-Yau $d$-folds the mirror map acts between different manifolds and their moduli spaces, torus and $K 3$ are "self-mirror" and accordingly the mirror map acts within the same moduli space [34].
} 


\subsection{PICARD FUCHS EQUATIONS, AND THEIR SYMMETRIC SQUARE}

We have seen in the preceding sections that a lot can be learned by simply focussing on the leading singularities in the moduli space of the effective theory. However, for the sake of performing really non-trivial quantitative tests of the heterotic/F-theory duality, we should try harder in order to reproduce the exact functional form of the couplings $\Delta_{\text {eff }}(T)$ from $K 3$ geometry. The hope is, of course, to learn something new about how to do exact non-perturbative computations in $D$-brane physics.

More specifically, the issue is to eventually determine the extra contributions $\delta(\lambda)$ to the geometric Green's functions in (1.24). Having a priori no good clue from first principles how to do this, the results of the previous section, together with experience with four dimensional compactifications with $N=2$ supersymmetry [35, 36, 37], suggest that somehow mirror symmetry should be a useful tool. It was indeed shown in refs. $[6,7]$ how mirror symmetry can be formally used to do such computations, although a satisfying physical insight why it works has not yet been achieved. We will therefore only briefly sketch the findings of these works, and refer the interested reader to them for more details.

The starting point is the observation that threshold couplings of similar structure appear also in four dimensional, $N=2$ supersymmetric compactifications of type II strings on Calabi-Yau threefolds. More precisely, these coupling functions multiply operators of the form $\operatorname{Tr} F_{G}{ }^{2}$ (in contrast to quartic operators in $d=8$ ), and can be written in the form

$$
\Delta_{\mathrm{eff}}^{(4 d)} \sim \ln \left[\lambda^{\alpha_{1}}(1-\lambda)^{\alpha_{2}}\left(\lambda^{\prime}\right)^{3}\right]+\gamma(\lambda)
$$

which looks similar to (1.24). By analyzing $[6,7]$ the known results of mirror symmetry computations $[36,37]$ in $d=4$, it is found that the "extra" term $\gamma(\lambda)$ in (1.37) appears also in the dilaton flat coordinate. That is, it is nothing but the remainder of the dilaton in the large dilaton limit: $S=-\ln [y]+\gamma$, where $y \sim e^{-S}+\ldots$ is a geometric coordinate of the underlying $\mathrm{CY}$ threefold moduli space.

The dilaton $S$ is a period associated with the CY threefold, and like all period integrals, it satisfies a system of linear differential equations. The idea $[6,7]$ is thus to first isolate a differential equation that is satisfied by $\gamma(\lambda)$ in $d=4$, and then to see how to generalize it such as to obtain a differential equation for $\delta(\lambda)$ in $d=8$. Furthermore, this differential equation may then be translated back into geometry, and this then would hopefully give us a clue about what the relevant quantum geometry is that underlies those quartic gauge couplings in eight dimensions. 
The starting point is, once again, the families of singular $K 3$ surfaces in (1.15). As we have seen above, associated with them are the period integrals (1.25), which evaluate to the hypergeometric functions given in (1.27). Generally, period integrals satisfy the "Picard-Fuchs" linear differential equations [38], and for our examples (1.15) these read: $\mathcal{L}_{N}$. $\varpi_{i}(z)=0$, where

$$
\mathcal{L}_{N}(z)=\theta_{z}^{2}-z\left(\theta_{z}+\frac{1}{2 N}\right)\left(\theta_{z}+\frac{1}{2}-\frac{1}{2 N}\right) .
$$

Here we have made for convenience a change of variables: $z \equiv-4 \lambda /(1-$ $\lambda)^{2}$; moreover, $\theta_{z} \equiv z \frac{\partial}{\partial z}$.

The four-dimensional theories are obtained by compactifying the type II strings on CY threefolds of special type, namely they are fibrations $[36,39]$ of the $K 3$ surfaces $(1.15)$ over $\mathbb{P}^{1}$. The size of the $\mathbb{P}^{1}$ yields then an additional modulus, whose associated flat coordinate is precisely the dilaton $S$ (in the dual, heterotic language; from the type II point of view, it is simply another geometric modulus). The $K 3$-fibered threefolds are then associated with enlarged PF systems of the form:

$$
\begin{aligned}
\mathcal{L}_{N}(z, y) & =\theta_{z}\left(\theta_{z}-2 \theta_{y}\right)-z\left(\theta_{z}+\frac{1}{2 N}\right)\left(\theta_{z}+\frac{1}{2}-\frac{1}{2 N}\right) \\
\mathcal{L}_{2}(y) & =\theta_{y}{ }^{2}-2 y\left(2 \theta_{y}+1\right) \theta_{y} .
\end{aligned}
$$

Since we are interested in the perturbative, one-loop contributions on the heterotic side (which capture the full story in $d=8$, in contrast to $d=4$ ), we need to consider only the weak coupling limit, which corresponds to the limit of large base space: $y \sim e^{-S} \rightarrow 0$. Though we might now be tempted to drop all the $\theta_{y} \equiv y \partial_{y}$ terms in the PF system, we better note that the $\theta_{y}$ term in $\mathcal{L}_{N}(z, y)$ can a non-vanishing contribution, namely in particular when it hits the logarithmic piece of the dilaton period, $S=-\ln [y]+\gamma$. As a result one finds that the piece $\gamma$ that we want to compute satisfies in the limit $y \rightarrow 0$ the following inhomogenous differential equation:

$$
\mathcal{L}_{N} \cdot\left(\gamma \varpi_{0}\right)(z)=\varpi_{0}(z)
$$

We now apply the inverse of this strategy to our eight dimensional problem. Since we know from the perturbative heterotic calculation of section 2 what the exact answer for $\delta$ must be (e.g., (1.3)), we can work backwards and see what inhomogenous differential equation the extra contribution $\delta(\lambda)$ obeys. What we find after some tedious computations is that it satisfies:

$$
\mathcal{L}_{N}^{\otimes 2} \cdot\left(\delta \varpi_{0}^{2}\right)(z)=\varpi_{0}^{2}(z)
$$


whose homogenous operator

$$
\mathcal{L}_{N}^{\otimes 2}(z)=\theta_{z}{ }^{3}-z\left(\theta_{z}+1-\frac{1}{N}\right)\left(\theta_{z}+\frac{1}{2}\right)\left(\theta_{z}+\frac{1}{N}\right),
$$

is the "symmetric square" [40, 41] of the $K 3$ Picard-Fuchs operator (1.38). This means that its solution space is given by the symmetric square of the solution space of $\mathcal{L}_{N}(z)$, i.e.,

$$
\mathcal{L}_{N}^{\otimes 2} \cdot\left(\varpi_{0}^{2}, \varpi_{0} \varpi_{1}, \varpi_{1}^{2}\right)=0 .
$$

Even though the inhomogenous PF equation (1.41) concisely captures the extra corrections in the eight-dimensional threshold terms, the considerations leading to this equation have been rather formal and it would be clearly desirable to get a better understanding of what it mathematically and physically means.

Note that in the four dimensional situation, the PF operator $\mathcal{L}_{N}(z)$, which figures as homogenous piece in (1.40), is by construction associated with the $K 3$ fiber of the threefold. By analogy, the homogenous piece of equation (1.41) should then tell us something about the geometry that is relevant in the eight dimensional situation. Observing that the solution space (1.43) is given by products of the $K 3$ periods, it is clear what the natural geometrical object is: it must be the symmetric square $\operatorname{Sym}^{2}(K 3)=(K 3 \times K 3) / \mathbb{Z}_{2}$. Being a hyperkähler manifold [42], its periods (not subject to world-sheet instanton corrections) indeed enjoy the factorization property exhibited by (1.43).
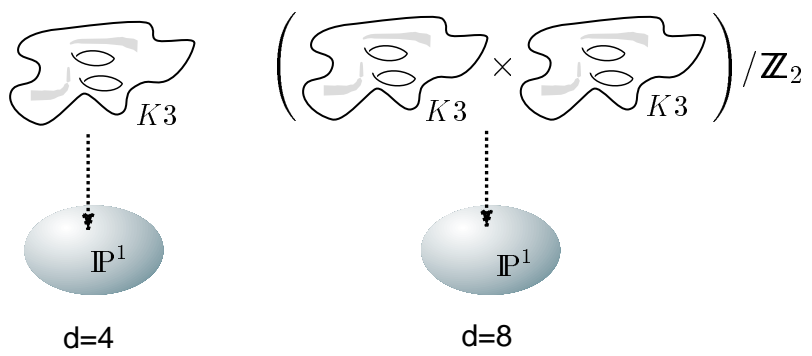

Figure 1.10 Formal similarity of the four and eight-dimensional string compactifications: the underlying quantum geometry that underlies the quadratic or quartic gauge couplings appears to be given by three- or five-folds, which are fibrations of $K 3$ or its symmetric square, respectively. The perturbative computations on the heterotic side are supposdly reproduced by the mirror maps on these manifolds in the limit where the base $\mathbb{P}^{1}$ 's are large.

The occurrence of such symmetric products is familiar in $D$-brane physics. The geometrical structure that is relevant to us is however not just the symmetric square of $K 3$, but rather a fibration of it, in 
the limit of large base space - this is precisely what the content of the inhomogenous PF equation (1.41) is. It is however not at all obvious to us why this particular structure of a hyperkähler-fibered five-fold would underlie the non-perturbative quantum geometry of the quartic gauge couplings in eight dimensions.

The situation is, in this respect, somewhat similar to $N=2 \mathrm{SYM}$ theory in four dimensions, where the Riemann surfaces underlying the effective lagrangian were found in [43], and at the time the geometry appeared to be merely a convenient mathematical tool for encoding appropriate physical data. It was only quite some time later when the geometry was given a deep physical interpretation.9 In the same spirit, one may speculate that the five-folds that seem to emerge here may ultimately have an interpretation in terms of a yet unknown dual formulation of the theory, or, perhaps more likely, in terms of sigma-models describing the relevant 7-brane interactions that lead to the requisite $F^{4}$ terms in the effective action.

\section{CONCLUSION}

Summarizing, we have seen that the postulated duality between the heterotic string compactified on $T^{2}$ and $F$-theory on $K 3$ (which is defined to be type IIB strings on $\mathbb{P}^{1}$ with $24(p, q)$ 7-branes) passes numerous tests. In particular, highly non-trivial quartic gauge threshold coupling functions, which are one-loop exact on the heterotic side, can be reproduced from geometrical data pertaining to $K 3$ surfaces. Still, a better physical understanding of the issues discussed in the previous paragraphs would be highly desirable.

\section{Acknowledgments}

I thank Stephan Stieberger and Nick Warner for the collaboration on this subject, and moreover the organizers of the School for a very pleasant stay.

${ }^{8}$ The same structure is found also when considering models with more moduli; in particular, the prepotential $\mathcal{G}(T, U)$ in (1.4) can be re-derived in an analogous fashion from symmetric squares [7].

${ }^{9}$ For example, as part of world-volumina of type IIA [44] or $M$-theory [45] fivebranes. 


\section{References}

[1] C. Vafa, Evidence for F-Theory, Nucl. Phys. B469 403 (1996), hep-th/9602022.

[2] C. Bachas, C. Fabre, E. Kiritsis, N.A. Obers and P. Vanhove, Heterotic/type-I duality and D-brane instantons, Nucl. Phys. B509 33 (1998), hep-th/9707126.

[3] E. Kiritsis and N.A. Obers, Heterotic type $I$ duality in $d<10$ dimensions, threshold corrections and D-instantons, JHEP10 004 (1997), hep-th/9709058.

[4] C. Bachas, Heterotic versus type I, Nucl. Phys. Proc. Suppl. 68348 (1998), hep-th/9710102;

M. Bianchi, E. Gava, F. Morales and K.S. Narain, D-strings in unconventional type I vacuum configurations, Nucl. Phys. B547 96 (1999), hep-th/9811013;

E. Gava, A. Hammou, J.F. Morales and K.S. Narain, On the perturbative corrections around D-string instantons, JHEP03 023 (1999), hep-th/9902202;

K. Foerger and S. Stieberger, Higher derivative couplings and heterotic-type I duality in eight dimensions, hep-th/9901020;

M. Gutperle, A note on heterotic/type I duality and D0 brane quantum mechanics, JHEP05 007 (1999), hep-th/9903010;

E. Gava, K.S. Narain and M.H. Sarmadi, Instantons in $N=2 S p(N)$ superconformal gauge theories and the AdS/CFT correspondence, hep-th/9908125.

[5] W. Lerche and S. Stieberger, Prepotential, mirror map and F-theory on K3, Adv. Theor. Math. Phys. 21105 (1998), hep-th/9804176.

[6] W. Lerche, S. Stieberger and N.P. Warner, Quartic gauge couplings from K3 geometry, hep-th/9811228. 
[7] W. Lerche, S. Stieberger and N.P. Warner, Prepotentials from symmetric products, hep-th/9901162.

[8] W. Lerche, Elliptic index and superstring effective actions, Nucl. Phys. B308 (1988) 102.

[9] C. Bachas and E. Kiritsis, $F^{4}$ terms in $N=4$ string vacua, Nucl. Phys. Proc. Suppl. 55B 194 (1997), hep-th/9611205.

[10] A. Schellekens and N. Warner, Anomalies, characters and strings, Nucl. Phys. B287 (1987) 317;

E. Witten, Elliptic genera and quantum field theory, Comm. Math. Phys. 109 (1987) 525;

W. Lerche, B.E.W. Nilsson, A.N. Schellekens and N.P. Warner, Nucl. Phys. 299 (1988) 91.

[11] J.A. Harvey and G. Moore, Algebras, BPS States, and Strings, Nucl. Phys. B463 315 (1996), hep-th/9510182.

[12] G. Lopes Cardoso, D. Lust and T. Mohaupt, Threshold corrections and symmetry enhancement in string compactifications, Nucl. Phys. B450 115 (1995), hep-th/9412209.

[13] B. de Wit, V. Kaplunovsky, J. Louis and D. Lust, Perturbative couplings of vector multiplets in $N=2$ heterotic string vacua, Nucl. Phys. B451 53 (1995), hep-th/9504006;

I. Antoniadis, S. Ferrara, E. Gava, K.S. Narain and T.R. Taylor, Perturbative prepotential and monodromies in $\mathrm{N}=2$ heterotic superstring, Nucl. Phys. B447 35 (1995), hep-th/9504034.

[14] P.S. Aspinwall, K3 surfaces and string duality, hep-th/9611137.

[15] J.H. Schwarz, An SL(2,Z) multiplet of type IIB superstrings, Phys. Lett. B360 13 (1995), hep-th/9508143.

[16] K. Kodaira, Ann. Math. 77 (1963) 563; Ann. Math. 78 (1963) 1.

[17] See eg.:

A. Johansen, A comment on BPS states in F-theory in 8 dimensions, Phys. Lett. B395 36 (1997), hep-th/9608186;

M.R. Gaberdiel and B. Zwiebach, Exceptional groups from open strings, Nucl. Phys. B518 151 (1998), hep-th/9709013.

M.R. Gaberdiel, T. Hauer and B. Zwiebach, Open stringstring junction transitions, Nucl. Phys. B525 117 (1998), hep-th/9801205;

O. DeWolfe and B. Zwiebach, String junctions for arbitrary 
Lie algebra representations, Nucl. Phys. B541 509 (1999), hep-th/9804210.

[18] A. Sen, F-theory and Orientifolds, Nucl. Phys. B475 562 (1996), hep-th/9605150.

[19] K. Dasgupta and S. Mukhi, F-theory at constant coupling, Phys. Lett. B385 125 (1996), hep-th/9606044.

[20] M. Green, J.A. Harvey and G. Moore, I-brane inflow and anomalous couplings on D-branes, Class. Quant. Grav. 1447 (1997), hep-th/9605033.

[21] K. Dasgupta, D.P. Jatkar and S. Mukhi, Gravitational couplings and $Z(2)$ orientifolds, Nucl. Phys. B523 465 (1998), hep-th/9707224.

[22] J.F. Morales, C.A. Scrucca and M. Serone, Anomalous couplings for D-branes and O-planes, Nucl. Phys. B552 291 (1999), hep-th/9812071.

[23] B. Craps and F. Roose, Anomalous D-brane and orientifold couplings from the boundary state, Phys. Lett. B445 150 (1998), hep-th/9808074;

B.J. Stefanski, Gravitational couplings of D-branes and O-planes, Nucl. Phys. B548 275 (1999), hep-th/9812088.

[24] L. Dixon, D. Friedan, E. Martinec and S. Shenker, The conformal field theory of orbifolds, Nucl. Phys. B282 (1987) 13;

M. Bershadsky and A. Radul, Conformal field theories with additional Z(N) symmetry, Sov. J. Nucl. Phys. 47 (1988) 363-369;

F. Ferrari and J. Sobczyk, Bosonic Field Propagators on Algebraic Curves, hep-th/9909173.

[25] W. Lerche and S. Stieberger, On the anomalous and global interactions of Kodaira 7-planes, hep-th/9903232.

[26] A. S. Schwarz, Field theories with no local conservation of the electric charge, Nucl. Phys. B208 (1982) 141.

[27] R. Dijkgraaf, H. Verlinde and E. Verlinde, Topological strings in $d<1$, Nucl. Phys. B352 59 (1991).

[28] S. Cecotti and C. Vafa, Topological-antitopological fusion, Nucl. Phys. B367 359 (1991). 
[29] R. Rankin, Modular forms and functions, Cambridge University Press.

[30] G. Lopes Cardoso, G. Curio, D. Lüst and T. Mohaupt, On the duality between the heterotic string and F-theory in 8 dimensions, Phys. Lett. B389 479 (1996), hep-th/9609111.

[31] B.R. Greene, A. Shapere, C. Vafa and S. Yau, Stringy Cosmic Strings And Noncompact Calabi-Yau Manifolds, Nucl. Phys. B337 1 (1990).

[32] M.C. Barrozo, Map of Heterotic and Type IIB Moduli in 8 Dimensions, hep-th/9909178.

[33] See e.g., Essays and mirror manifolds, (S. Yau, ed.), International Press 1992; Mirror symmetry II, (B. Greene et al, eds.), International Press 1997.

[34] P.S. Aspinwall and D.R. Morrison, String theory on K3 surfaces, hep-th/9404151.

[35] S. Kachru and C. Vafa, Exact results for $N=2$ compactifications of heterotic strings, Nucl. Phys. B450 69 (1995), hep-th/9505105.

[36] A. Klemm, W. Lerche and P. Mayr, K3 Fibrations and heterotic type II string duality, Phys. Lett. B357 313 (1995), hep-th/9506112.

[37] S. Kachru, A. Klemm, W. Lerche, P. Mayr and C. Vafa, Nonperturbative results on the point particle limit of $N=2$ heterotic string compactifications, Nucl. Phys. B459 537 (1996), hep-th/9508155.

[38] S. Hosono, A. Klemm, S. Theisen and S.T. Yau, Mirror symmetry, mirror map and applications to Calabi-Yau hypersurfaces, Commun. Math. Phys. 167301 (1995), hep-th/9308122.

[39] P.S. Aspinwall and J. Louis, On the Ubiquity of K3 Fibrations in String Duality, Phys. Lett. B369 233 (1996), hep-th/9510234.

[40] B.H. Lian and S. Yau, Arithmetic properties of mirror map and quantum coupling, Commun. Math. Phys. 176163 (1996), hep-th/9411234, Mirror maps, modular relations and hypergeometric series I, hep-th/9507151, Mirror maps, modular relations and hypergeometric series. II, Nucl. Phys. Proc. Suppl. 46 248 (1996), hep-th/9507153. 
[41] M. Lee, Picard-Fuchs equations for elliptic modular varieties, Appl. Math. Letters 4, no.5 (1991) 91-95;

C. Doran, Picard-Fuchs Uniformization: Modularity of the Mirror Map and Mirror-Moonshine, math.AG/9812162.

[42] See e.g., D. Huybrechts, Compact hyperkähler manifolds: basic results, alg-geom/9705025;

R. Dijkgraaf, Instanton strings and hyperKähler geometry, hep-th/9810210.

[43] N. Seiberg and E. Witten, Electric - magnetic duality, monopole condensation, and confinement in $N=2$ supersymmetric Yang-Mills theory, Nucl. Phys. B426 19 (1994), hep-th/9407087.

[44] A. Klemm, W. Lerche, P. Mayr, C. Vafa and N. Warner, Self-Dual Strings and N=2 Supersymmetric Field Theory, Nucl. Phys. B477 746 (1996), hep-th/9604034.

[45] E. Witten, Solutions of four-dimensional field theories via M-theory, Nucl. Phys. B500 3 (1997), hep-th/9703166. 